\title{
Resting-State Functional Connectivity Disruption as a Pathological Biomarker in Autosomal Dominant Alzheimer Disease
}

\begin{abstract}
Robert X Smith $\mathrm{PhD}^{1}$, Jeremy F. Strain $\mathrm{PhD}^{1}$, Aaron Tanenbaum $\mathrm{BA}^{1}$, Anne M. Fagan $\mathrm{PhD}^{1}$, Jason Hassenstab PhD ${ }^{1}$, Eric McDade DO ${ }^{1}$, Suzanne E. Schindler MD, PhD ${ }^{1}$, Brian A. Gordon $\mathrm{PhD}^{2}$, Chengjie Xiong $\mathrm{PhD}^{3}$, Jasmeer Chhatwal MD, $\mathrm{PhD}^{4}$, Clifford Jack Jr MD ${ }^{5}$, Celeste Karch $\mathrm{PhD}^{10}$, Sarah Berman MD, PhD ${ }^{6}$, Jared R. Brosch $\mathrm{MD}^{7}$, James J. Lah MD, PhD ${ }^{8}$, Adam Brickman $\mathrm{PhD}^{9}$, David M Cash MD ${ }^{11}$, Nick C. Fox MD ${ }^{11}$, Neill R Graff-Radford MD ${ }^{12}$, Johannes Levin $\mathrm{MD}^{13}$, James Noble MD ${ }^{9}$, David M. Holtzman MD ${ }^{1}$, Colin L. Masters MD ${ }^{14}$, Martin R. Farlow MD ${ }^{7}$, Christoph Laske ${ }^{16}$, Peter R. Schofield MD ${ }^{15}$, Daniel S. Marcus PhD ${ }^{2}$, John C. Morris MD ${ }^{1}$, Tammie L. S. Benzinger MD, PhD², Randall J. Bateman MD, Beau M. Ances MD, PhD ${ }^{1}$ for the DIAN Network
\end{abstract}

${ }^{1}$ Department of Neurology, Washington University in Saint Louis, St. Louis, MO 63110

${ }^{2}$ Department of Radiology, Washington University in Saint Louis, St. Louis, MO 63110

${ }^{3}$ Department of Biostatistics, Washington University in Saint Louis, St. Louis, MO 63110

${ }^{4}$ Department of Neurology, Massachusetts General Hospital, Boston, MA, 02114

${ }^{5}$ Department of Radiology, Mayo Clinic, Rochester, MN, 55905

${ }^{6}$ Department of Neurology, University of Pittsburgh, Pittsburgh, PA, 15260

${ }^{7}$ Department of Neurology, Indiana University, Indianapolis, IN, 46202

${ }^{8}$ Department of Neurology, Emory University, Atlanta, GA, 30329

${ }^{9}$ Department of Neurology, Columbia University, New York, NY, 100310

${ }^{10}$ Department of Psychiatry, Washington University School of Medicine, St. Louis, MO, 63110.

${ }^{11}$ Dementia Research Centre, Department of Neurodegenerative Disease, Institute of Neurology, University College London, London, UK.

${ }^{12}$ Department of Neurology, Mayo Clinic, Jacksonville, FL, USA

${ }^{13}$ German Center for Neurodegenerative Disease (DZNE) Munich, Munich, Germany 
${ }^{14}$ The Florey Institute, University of Melbourne, Parkvile, VIC, Australia

${ }^{15}$ Neuroscience Research Australia, Sydney, NSW, Australia

${ }^{16}$ Eberhard Karls University of Tubingen, Tubingen Germany

${ }^{17}$ Hope Center for Neurological Disorders, Knight ADRC, Washington University, St. Louis, MO, USA

Brief Running Title: Functional Connectivity Disruption in Autosomal Dominant Alzheimer Disease

Abstract Word Count: 250

Manuscript Word Count: 4,655 
Aim: Identify a global resting state functional connectivity (gFC) signature in mutation carriers (MC) from the Dominantly Inherited Alzheimer Network (DIAN). Assess the gFC with regards to amyloid $(\mathrm{A})$, tau $(\mathrm{T})$, and neurodegeneration $(\mathrm{N})$ biomarkers and estimated years to symptom onset (EYO).

Introduction: Cross-sectional measures were assessed in $M C(n=171)$ and mutation noncarriers (NC) $(n=70)$ participants. A FC matrix that encompassed multiple resting state networks (RSNs) was computed for each participant.

Methods: A global FC was compiled as a single index indicating functional connectivity strength. The gFC signature was modeled as a non-linear function of EYO. The gFC was linearly associated with other biomarkers used for assessing the AT(N) framework including: cerebrospinal fluid (CSF), positron emission tomography (PET) molecular biomarkers, and structural magnetic resonance imaging.

Results: The gFC was reduced in MC compared to NC participants. When MC participants were differentiated by clinical dementia rating (CDR), the gFC was significantly decreased in MC CDR > 0 (demented) compared to either MC CDR 0 (cognitively normal) or NC participants. The gFC varied non-linearly with EYO and initially decreased at EYO $=-24$ years, followed by a stable period followed by a further decline near EYO $=0$ years. Irrespective of EYO, a lower gFC associated with values of amyloid PET, CSF A $\beta_{1-42}$, CSF ptau, CSF t-tau, FDG, and hippocampal volume.

Conclusions: The gFC correlated with biomarkers used for defining the AT(N) framework. A biphasic change in the gFC suggested early changes associated with CSF amyloid and later changes associated with hippocampal volume.

Key words: Autosomal dominant Alzheimer disease, resting state functional connectivity, estimated years to onset (EYO), cerebrospinal fluid (CSF), positron emission tomography (PET), amyloid, tau, fluorodeoxyglucose (FDG), hippocampus, 
Impact Statement

This project focused on creating and evaluating a global FC signature that may serve as an outcome measure in clinical trials. This global FC signature encompassed multiple resting state networks (RSNs) that included both inter- and intra-networks. Prior studies that focus on a single network may overlook important changes seen within and between networks. Our analysis is a logical progression from previous work that demonstrated that intra- and inter-network brain connections across multiple networks were affected with progression to cognitive impairment in ADAD. This work revealed that FC disruption exhibits a nonlinear time course that was consistent with proposed biomarker models. 


\section{INTRODUCTION}

Alzheimer disease (AD) is the leading cause of dementia in the United States. The occurrence of $A D$ has been linked with $\beta$-amyloid $(A \beta)$ peptide aggregating into plaques in the brain [1]. A $\beta$ levels vary naturally following a circadian rhythm [5] and are associated with neuronal excitability [6]. Excess production from neuronal hyper-excitability may underlie plaque formation [6, 6a]. The amyloid cascade hypothesis [7] proposes accumulation of $A \beta$ plaques results in neurotoxic effects that are associated with tau accumulation and neurodegeneration culminating in cognitive dysfunction (as assessed by the clinical dementia rating (CDR)) [8]. Autosomal dominant Alzheimer disease (ADAD) is caused by $\sim 100 \%$ penetrant mutations in genes that encode for the amyloid precursor protein (APP) [2-4] or gamma-secretase components, presenilin (PSEN) 1 and PSEN2. The ability to estimate when mutation positive (MC) carriers will develop cognitive changes allows for modeling of disease based on estimated years to symptom onset (EYO). ADAD typically occurs at an earlier age compared to late-onset AD (LOAD) [9-11] and is not associated with age-related comorbidities.

Pathologic changes start decades before clinical symptoms manifest in ADAD [8]. Recently, a set of biomarker criteria has been proposed to stage late onset AD disease progression [11a]. This model describes the earliest changes involve amyloid accumulation (A), followed by tau $(T)$ deposition, and eventually neurodegeneration $(N)$ that lead to cognitive dysfunction [12-19]. Changes in amyloid biomarkers, such as cerebrospinal fluid (CSF) $A \beta_{1-42}$ and [11C] Pittsburgh compound $B(P i B)$ amyloid positron emission tomography (PET), have been observed 15-20 years before symptom onset in $\operatorname{ADAD}[8,18,20,21,22$, 23]. Tau (T) biomarkers, such as CSF phosphorylated tau ${ }_{181}\left(p-\operatorname{tau}_{181}\right)$ [4], change $\sim 10-15$ years prior to symptom onset in ADAD. Neurodegeneration (N) biomarkers, such as CSF total tau (t-tau) [4, 23] 18F-fluorodeoxyglucose (FDG) PET, a measure of glucose metabolism [7], and magnetic resonance imaging (MRI) measurements of hippocampal volume $[8,22,23,24,25]$, change at $\sim 5-10$ years prior to EYO. CSF-based measures are sensitive to global levels of amyloid accumulation and tau deposition, but lack information pertaining to what brain regions are affected. Imaging, on the other hand, provides spatially detailed information regarding impacted brain regions. In fact, PET imaging has 
revealed amyloid may accumulate in a pattern of brain regions distributed across multiple functional brain networks [70]. This has led some to hypothesize that pathological spread of disease through the brain occurs via brain networks [26, 70, 71]. However, a gap persists regarding when changes in brain networks occur with respect to AT(N) biomarkers in ADAD.

Resting-state functional connectivity (FC) non-invasively measures the association of signaling among brain regions and can be used to identify resting state networks (RSN) [27]. The inter-relationships among RSNs is sensitive to neuronal dysfunction and is associated with the degree of cognitive impairment [28-30]. A reduction in both intra- and inter-network FC occurs in ADAD [31]. Global metrics of amyloid and tau accumulation aggregate spatially selective patterns for increased sensitivity. Similarly, a global FC signature can be derived from a combination of changes in intra- and inter-networks that spans multiple RSNs $[53,69]$. It remains important to know where changes in this global FC signature occur in the temporal progression of ADAD.

The purpose of this study is to evaluate the role of FC within the AT(N) framework in ADAD. Prior work has focused on select FC network differences however, we employ a data reduction strategy to compile information across multiple intra- and inter-network connections into a single global FC signature of ADAD. We assess FC strength based on a global FC signature between NC, MC asymptomatic (CDR 0), and MC symptomatic (CDR > $0)$. We also evaluate the relationship between the global FC signature and established biomarkers that comprise the AT(N) framework and model the global FC signature as a function of EYO.

\section{METHODS}

\section{Participant characteristics:}

The cohort consisted of 171 individuals with ADAD mutations (MC) and 104 individuals from $A D A D$ families who were genetically at-risk for inheriting the mutation but were not carriers (NC). All participants were enrolled in the international Dominantly Inherited Alzheimer Network (DIAN) and extracted from Data Freeze 11. Inclusion into this current analysis required that participants complete a general physical (including neurologic) 
examination, health and medication history, clinical assessment for dementia [32], biochemical analysis, neuroimaging acquisition on only 3T Siemens Tim Trio scanners (Erlangen, Germany), and successful pass all quality control criteria (e.g. reduced motion during neuroimaging acquisition). All participants or their representatives provided written informed consent that was in accordance with the Washington University institutional review boards or their respective institutions provided approval.

\section{Clinical Dementia Rating (CDR):}

Experienced clinicians conducted semi-structured interviews of each participant and a knowledgeable collateral source. The CDR was used to evaluate degree of impairment [32]. A score of CDR 0 indicates cognitively normal, CDR 0.5 corresponds to very mild dementia, and $C D R \geq 1$ specifies mild to moderate dementia. Participants with a score of CDR $>0$ had a clinical diagnosis of AD dementia using previously described criteria [33].

\section{EYO estimation:}

Parent age at symptomatic onset was determined from semi-structured interviews with the participant, a knowledgeable collateral source, and/or other informants familiar with the parental history of disease. The age at onset of the affected parent was determined by estimating the time of onset of consistent symptoms (e.g. memory/cognition, motor, or behavior) [2]. The EYO for each individual from DIAN was defined as age at testing minus the age at symptom onset for that individual's affected parent [34].

\section{Biochemical analyses:}

A lumbar puncture was performed in the morning under fasting conditions to obtain CSF using previously described methods [8]. Samples were shipped on dry ice to the DIAN biomarker core laboratory. CSF concentrations of $A \beta_{1-42}, t$-tau, and $p$-tau at threonine 181 ( $p$-tau $\left.{ }_{181}\right)$ were measured by immunoassay (INNOTEST A $\beta_{1-42}$ and INNO-BIA AlzBio3, Innogenetics). All values met quality-control standards, including a coefficient of variation of $25 \%$ or less and kit "controls" that were within the expected range as defined by the manufacturer. Measurement consistency between plates of a common sample were included in each run. 
Imaging:

MRI data from only 3T Siemens Tim Trio scanners (Erlangen, Germany) were analyzed. Scanners were calibrated and used similar protocols. Structural images were acquired using the Alzheimer's Disease Neuroimaging Initiative (ADNI) protocol. T1-weighted scans were automatically segmented into regions of interest according to the Desikan atlas through FreeSurfer (Martinos Center for Biomedical Imaging, Charlestown, Massachusetts, USA). Blood oxygen level-dependent (BOLD) FC was also acquired using previously described methods [31]. During the BOLD FC scans, participants were instructed to remain still with their eyes open and not fall asleep.

PET imaging was performed using FDG to measure glucose metabolism and PiB to measure amyloid load. PET imaging analyses were performed using a previously described PET Unified Pipeline (PUP) [36]. Data from the 40 to 70 minute post-injection window for PiB and 40 to 60 minute window for FDG were converted to standardized uptake value ratios (SUVR) in the defined regions of interest using the cerebellar cortex as a reference region [37]. Partial volume correction (PVC) was performed with a regional spread function (RSF) [38] that uses a geometric transfer matrix (GTM) technique [39]. Global A $\beta$ was summarized as the average SUVR for the precuneus, lateral temporal, gyrus rectus and prefrontal regions that have previously been shown to delineate $A D$ from cognitively normal controls [36]. FDG from the precuneus was utilized as changes in this region are reliable and occur early in DIAN participants $[40,22,23]$.

\section{FC Preprocessing:}

BOLD FC preprocessing followed previously described methods $[20,30,31]$ including correction of odd versus even slice intensity differences attributable to interleaved acquisition and compensation for head movement within and across runs. Intensity inhomogeneity was corrected using FSL FAST [41] followed by intensity normalization to obtain a whole brain mode value of 1000. Echoplanar imaging (EPI) distortion due to magnetization inhomogeneity was corrected using a mean field map [42]. Atlas transformation was computed by registering the EPI mean image to an atlasrepresentative template via the MP-RAGE (EPI $\rightarrow$ MP - RAGE $\rightarrow$ template). The template 
was generated from a separate cohort of 12 cognitively normal individuals. Compensation for head motion, distortion correction, and atlas transformation were sequentially combined to generate a volumetric time series that was resampled in $3 \mathrm{~mm}^{3}$ atlas space.

Frames corrupted by excessive head motion were identified on the basis of both DVARS and frame displacement (FD) measures [43]. In greater detail, the DVARS criterion was $0.9 \%$ root mean square $(\mathrm{rms})$ of the frame-to-frame signal change from the entire brain $[44,45]$. The FD criterion was $0.3 \mathrm{~mm}$. Frames were censored if either criterion was exceeded. The time series were band-pass filtered to retain frequencies between $0.005 \mathrm{~Hz}$ and $0.1 \mathrm{~Hz}$. For purposes of filtering, only a linear interpolation was applied over censored frames. Censored frames were excluded from all subsequent steps.

Denoising was accomplished using a CompCor-like strategy [46]. In brief, nuisance regressors were derived from three compartments (white matter, ventricles and the extraaxial space) and then dimensionality-reduced to create a matrix for singular value decomposition (SVD). White matter and ventricle masks were segmented in each individual using FreeSurfer 5.3 [47] and spatially resampled to register with the FC data. Time series also were extracted from high-variance voxels (temporal standard deviation $>2.5 \%$ relative to the whole brain mode) in the extra-axial space (excluding the eyes). Nuisance regressors were derived from white matter, ventricles, and the extra-axial space. The final set of nuisance regressors included six parameters derived from rigid body headmotion correction, the global signal (GS) averaged over the (FreeSurfer-segmented) brain, and the GS temporal derivative. The preprocessed time series was non-linearly warped to Montreal Neurological Institute (MNI) 152 space ( $3 \mathrm{~mm}^{3}$ voxels) space using FNIRT [48-51].

\section{FC post-processing}

A putative set of 246 functional regions-of-interest (ROI) were organized into 12 RSNs that included the sensorimotor (SM) sensorimotor-lateral (SMlat), cingulo-opercular (CO), auditory (AUD), ventral attention (VAN), visual (VIS), salience (SAL), default mode (DMN), memory (MEM), dorsal attention (DAN), subcortical (SUB), and frontoparietal (FP) [39]. ROIs were defined as $10 \mathrm{~mm}$ diameter spheres whose center coordinates were in MNI atlas space [52]. All ROI's were distinct and occupied unique voxels. A 246× FC matrix was 
obtained for each individual as follows. First, the pre-processed FC data was transformed on to the MNI atlas. Second, the mean time series was computed within each ROI excluding the censored frames. The pairwise correlation between all ROI time series was then derived. Correlation values were Fisher transformed for normality. The mean $246 \times 246$ FC matrix across all participants is shown in Figure 1 (left). We used each ROI's RSN designation [52] to compute the average intra- and inter-network correlation reducing the $\mathrm{FC}$ to a $12 \times 12$ matrix. FC values along the diagonal blocks represent intra-RSN correlations, and values in the off diagonal blocks represent inter-RSN correlations. The mean $12 \times 12 \mathrm{FC}$ matrix across all participants is shown in Figure 1 (middle).

\section{FC Principal Components Analysis (PCA)}

Data reduction was performed to isolate a global FC signature metric of global FC changes $[53,69]$. The intra- and inter-network pattern of FC values from each participant were compiled, and a single global FC signature was selected. Specifically, the 12 intra-network and $(12 \times(12-1)) / 2=66$ inter-network averages (total of $12+66=78$ ) were compiled from all participants $(n=275)$ into a single $275 \times 78$ matrix. A PCA of this matrix was performed by singular value decomposition (SVD). PCA is a multivariate analysis that reveals internal data organization and its variance. This PCA revealed distinct FC patterns of intra- and inter-network averages across all RSNs. Each pattern is comprised of weights describing the influence from each of the 78 intra- and inter-network FC composites on that pattern. The magnitude of a given weight reflects the strength of influence while the sign, positive or negative, indicates whether the corresponding intra- or inter-network FC composite tends to increase or decrease, respectively, across participants. Each pattern was also accompanied by a set of scores that represented its association with each participant's pattern of FC values. The variability of these scores related to the total variability of FC values among participants. A low score indicates the primary pattern is weakly present or absent from a participant. This implicates a strong deviation from, or decline in, the network connectivity specified by the primary pattern. The primary pattern that captured the largest percentage of total inter-individual FC variance $(21 \%)$ was designated the global FC signature for ADAD (Figure 1, right). The same data reduction was applied to NC participants. The primary pattern from NC was very similar to the pattern computed for all 
participants, indicating patient characteristics (abnormal levels of $A \beta_{42}$, tau, and neurodegeneration) are not strong factors in determining the primary pattern.

\section{EYO modeling}

\section{Global FC signature versus RSN}

We compared the abilities of the global FC signature and intra-network values of the 12 RSNs included in this study to, separately, predict EYO using a leave-one-out crossvalidation (LOOCV). Specifically, for each FC metric:

1. FC and EYO data were split into two sets: train, and test. The training set comprised of data from N-1 mutation positive participants, and a test set comprising data from a single mutation positive participant.

2. A linear predictive model was computed using the training set:

$$
\mathrm{EYO}_{\text {train }}=\beta_{0}+\beta_{1} \mathrm{FC}_{\text {train }}
$$

3. The linear model (i.e. coefficients $\beta_{0}$ and $\beta_{1}$ ) was used to predict EYO of the test set:

$$
\mathrm{EYO}_{\text {pred }}=\beta_{0}+\beta_{1} \mathrm{FC}_{\text {test }}
$$

4. The error in EYO was computed:

$$
\mathrm{EYO}_{\text {err }}=\mathrm{EYO}_{\text {test }}-\mathrm{EYO}_{\text {pred }}
$$

5. Steps 1- 4 were repeated such that the FC data for each mutation positive participant was used in the test set.

6. The sum of squares (SS) was computed for both EYOerr and EYO:

$$
\begin{gathered}
\mathrm{SS}_{\text {err }}=\sum\left(\mathrm{EYO}_{\text {err }}\right)^{2} \\
\mathrm{SS}_{E Y O}=\sum(\mathrm{EYO})^{2}
\end{gathered}
$$

7. The coefficient of determination $\left(R^{2}\right)$, the proportion of the variance in actual EYO that is predictable from the FC, was computed:

$$
R^{2}=1-\frac{S S_{\text {err }}}{S S_{E Y O}}
$$


Nonlinear modeling

$A D$ biomarkers exhibit nonlinear time courses across disease progression [8, 60, 60a]. Proposed biomarker models suggest nonlinear time courses with sigmoidal shape [60]. The sigmoid transition time (i.e., when peak rate-of-change occurs) varies based on the biomarker. We investigated a sigmoidal shape of the global FC signature for MC participants to estimate transition times. An unweighted moving average of the global FC signature for MC participants was computed as a function of EYO using bin sizes of \pm 5 years to capture long-term trends. A model of staggered logistic decline evaluated the relationship between the global FC signature and EYO (t) for MC participants:

$$
\text { global FC signature }=\mathrm{A}\left(\frac{1}{1+e^{-r\left(t-t_{1}\right)}}-\frac{1}{1+e^{-r\left(t-t_{2}\right)}}\right)
$$

where $A$ is carrying capacity, $r$ is growth rate, and $t_{1}$ and $t_{2}$ are transition times. Here the carrying capacity describes the maximum FC that can be sustained. The growth rates and carrying capacities for both curves were constrained by Goodness-of-fit using a ShapiroWilks test of normality on the residuals (57). Nonlinear least-squares regression was performed using the ' $\mathrm{nlm}$ ' function in R [54-56]. The Akaike Information Criterion (AIC), Bayesian Information Criterion, and Levene's test were used to further evaluate model performance by comparing to polynomial models (i.e. linear, quadratic, and cubic) [58].

\section{$\underline{\text { Association between global FC signature and AD biomarkers }}$}

Associations were computed between a participant's global FC signature and $A / T /(N)$ biomarkers including: CSF biomarkers (log transformed $A \beta_{1-42}(A 1), p-\operatorname{tau}_{181}(T 1)$, and totaltau (N1) ), PET molecular biomarkers (PiB mean cortical SUVR (A2) and FDG uptake in the precuneus (N2)), and a structural MRI biomarker (hippocampal volume (N3)). To investigate the relationship between global FC signature and AD biomarkers while adjusting for mediating associations, the partial correlation matrix $(P)$ was computed by matrix inversion of the correlation matrix $(R)$ such that the partial correlation between the $\mathrm{i}$-th and $\mathrm{j}$-th biomarker was defined as:

$$
p_{i j}=-\frac{s_{i j}}{\sqrt{s_{i i} s_{j j}}}
$$

where $s_{\text {_ }} \mathrm{ij}$ were the elements of the inverted correlation matrix $S=R^{\wedge}(-1)$. 


\section{RESULTS}

\section{Demographics}

Cross-sectional FC data was obtained for NC $(n=104)$ and MC $(n=171)$ participants. NC individuals were older than MC CDR $0(p<0.05)$ participants, but younger than MC CDR>0 $(p<0.05)$ participants. Similarly, MC CDR 0 participants were younger than MC CDR $>0$ participants $(p<0.05)$ (Table 1$)$.

\section{Spatial topology of the global FC signature of ADAD}

We observed a global FC signature of ADAD, with strong contributions from both intraand inter-network connections that spanned multiple RSNs (Figure 1). This global FC signature was influenced by both positive and negative correlations and, for visual clarity, was separated along the diagonal based on the direction of influence on the global FC signature. Networks whose positive correlations had a strong influence included the SM, SMlat, CO, AUD, VIS, and MEM (intra-network). Networks whose negative correlations had a strong negative influence included the FP, MEM (inter-network), DAN, and DMN.

\section{The global FC signature associates with markers of disease progression}

The global FC signature decreased with pathology. We observed the global FC signature was significantly decreased in MC (yellow) compared to NC (gray) participants (Figure 2A). When MC participants were further differentiated by CDR status, the global FC signature significantly decreased in MC CDR $>0$ (Figure $2 \mathrm{~A}$, red; $p<0.05$ ) compared to either $M C$ CDR 0 (green) and NC (gray) participants. NC and MC CDR 0 participants were not significantly different $(p>0.05)$. For MC participants a negative association was observed between mutation EYO and the global FC signature (Spearman's $\rho=-0.33, p=1.2 \mathrm{e}-05$;

Figure 2B). The association between the global FC signature and EYO remained significant after controlling for CDR status. We also observed that the global FC signature predicted, on average, 5 to 18 percent more variance for the actual EYO than inter-network RSN values (Figure $2 \mathrm{C}$ ). 
With regards to EYO, the global FC signature varied non-linearly across the time course of the disease (Figure 2D). This was consistent with a dual logistic behavior model characterized by two transition times that were offset by $\sim 17$ years (Shapiro-Wilk's W = $0.97, p=0.28)$. This nonlinear model $(\mathrm{AIC}=-161, \mathrm{BIC}=-153)$ performed significantly better than first $(A I C=-128, B I C=-123)$, second $(A I C=-128, B I C=-121)$, and third $(A I C=-126, B I C$ $=-118$ ) order polynomial models (Levene's $F=4.04, p=0.002$ ) and suggested a two stage process. The global FC signature was elevated early in the disease time course (EYO <-16.7 years) in $\mathrm{MC}$ followed by a period that resembled control levels until further decreasing near the estimated time of symptom onset ( $E Y O=0.5$ years).

\section{Association between global FC signature and biomarkers}

Strong associations were observed between CSF total tau and CSF $p$-tau $\tan _{181}(r=0.86, p<$ 10E-16), mean cortical PiB SUVR and CSF total tau ( $r=0.53, p=1.7 \mathrm{E}-9)$, mean cortical PiB SUVR and CSF $p-\operatorname{tau}_{181}(r=0.65, p=2.2 \mathrm{E}-16)$, and precuneus PET FDG and hippocampal volume $(r=0.53, p=1.6 \mathrm{E}-09)$.

Strong associations were observed between the global FC signature and several AD biomarkers (Figure 3B, left). The global FC signature was compared to each AD biomarker classified according to the amyloid $(\mathrm{A})$, tau $(\mathrm{T})$, and neurodegeneration $(\mathrm{N})$ framework (Figure $3 \mathrm{~A}$ ). With regards to A criteria, the global FC signature was negatively associated with amyloid deposition as measured by mean cortical PiB SUVR ( $p=0.03)$, and was positively correlated with CSF $A \beta_{1-42}(p=0.007)$. With regards to $T$ criteria, the global FC signature was negatively associated with CSF $p-\operatorname{tau}_{181}(p=0.009)$. With regards to $(N)$ criteria, the global FC signature was negatively associated with CSF total tau $(p=0.02)$, and positively associated with both precuneus PET FDG $(p=0.002)$ and hippocampal volume ( $p$ $=0.001$ ). For each of these biomarkers, a worse global FC signature score was associated with greater pathology.

To investigate the strength of the direct pathological relationship between any two biomarkers (including the global FC signature) we controlled for potential mediating effects of general pathological decline. Specifically, an unbiased model was computed 
using a partial correlation matrix that controlled for other biomarkers (Figure 3B, middle). In this unbiased model, no assumption of a specific temporal sequence of biomarkers was included. This network model strongly differentiated AT(N) biomarkers and agreed with previous hypothesized trajectories with amyloid measures strongly associating with each (A1 and A2), measure of tau (T1) strongly tied to neurodegeneration (N1) as well as measures of neurodegeneration group together (N1, N2, and N3) (Figure 3B, right). In this model, the global FC signature most strongly associated with CSF A $\beta_{1-42}(A 1)$ and hippocampal volume (N3). These results provide further evidence of a two-stage process concerning the global FC signature with changes associating with early and late biomarkers. Moreover, these results suggest each stage may be associated with changes in either amyloid accumulation or volumetrics.

\section{DISCUSSION}

These results provide evidence that disruption of multiple functionally connected brain networks occurs in ADAD mutation carriers that is both stage dependent (asymptomatic versus symptomatic) and state dependent (biomarker status). Disruption of the global FC signature was greatest in symptomatic MC carriers. This indicates that changes in the global FC signature are tightly coupled with clinical presentation. However, even after adjusting for symptom severity using CDR, disruption of the global FC signature was significantly associated with disease progression, as measured by EYO. Further, the global FC signature was, on average, a better predictor of EYO compared to intra-network values of individual RSNs. Changes in the global FC signature were also associated with pathological biomarkers classified using the $A T(N)$ framework for designating temporal progression of $A D$. Two independent methods showed that disruption of the global FC signature: 1) occurred primarily during early (EYO -17), and late (EYO 0) stages, and 2) was associated with early (CSF $A \beta_{1-42}$ ), and late (hippocampal atrophy) stage biomarker changes. Taken together, these results suggest the global FC signature may be sensitive to

distinct processes affecting synaptic activity: $A \beta$ accumulation early on in disease progression, and neurodegeneration during later stages. 
Our results are consistent with previous FC studies that investigated the disruption of specific RSNs in ADAD, but also sheds new light on patterned changes across the entire spatial topography. Previous studies have primarily focused on FC changes within a single network. Changes have typically focused on the DMN, as this network has been associated with amyloid deposition $[28,29]$ and is one of the largest networks with regards to overall size in the brain [27]. In our current analysis, a global FC pattern of disruption was observed that included both intra and inter-network brain connections. These results suggest that prior studies primarily focusing on changes within a single network may overlook important changes seen within not only a network but also changes between networks. Our analysis is a logical progression from previous work that demonstrated that intra- and inter-network brain connections across multiple networks were affected with progression to cognitive impairment in ADAD [31]. In our current analysis, RSNs that associated with cognitive impairment included cognitive processing networks including MEM, FP, DAN, and DMN as well as sensory cortical regions such as the SM, SM-lat, VIS, and AUD. Cognitive processing regions that have been associated with the changes in pathological biomarkers, such as amyloid and tau accumulation and volume loss, and disruption of FC in these regions, may be associated with positivity of one or more biomarkers. Interestingly, neither FC nor pathological changes are typically observed in primary sensory regions. These results suggest the FC disruption observed may be a precursor to subsequent pathology.

Disruption was greatest for symptomatic mutation carriers ( $M C$ CDR $>0$ ) compared to mutation non-carriers (NC). Our data showed that the greatest changes in the FC signature occurred for the symptomatic disease stage based on clinical staging and genetic profile. Specifically, our data showed that 50\% of the MC symptomatic cohort overlapped with the worst $25 \%$ of the asymptomatic MC cohort. This degree of overlap can be attributed to the classification scheme that is based on subjective responses during the clinical interview. This sensitivity to symptom manifestation bolsters the capability of this imaging marker as a tool for disease conversion. Future studies may assess the capability of an FC signature to redefine group classification to better identify individuals on the cusp of conversion. 
using linear modeling, but also provide new insight regarding the timeline of FC disruption. Linear modeling of disease progression suggests FC disruption begins prior to the onset of symptoms. Consistent with previous results, we used a linear model and observed that FC disruption in certain RSNs occurred approximately five years prior to expected symptom inset (EYO -5) [59]. However, previous reports show AD biomarkers exhibit nonlinear time courses across disease stages $[8,60]$. In an updated model of disease progression, we observed that the global FC signature also exhibited a nonlinear time course that was consistent with proposed biomarker models [60]. Specifically, disruption of the global FC signature was marked by two dramatic changes that occurred during very early ( 17 EYO) and late ( $\sim \mathrm{EYO})$ disease stages in $A D$ progression. The time period leading up to the initial decrease of the global FC signature could suggest a very early period of hyper-connectivity [63]. The production of $A \beta$ has been linked to increased synaptic activity that can manifest as non-convulsive seizures that are present prior to cognitive symptoms [60, 64-66]. FC hyperactivity has also been observed in LOAD particularly in areas with advanced $A B$ accumulation [67]. It remains unclear whether this hyperactivity is detrimental due to synaptic excitotoxicity or compensatory due to increased pathological burden [68]. Our data suggests this hyperactivity in FC may be a compensatory mechanism to preserve cognitive stability provided this observed state was years before symptom onset [69]. However, prolonged hyperactivity can lead to cognitive deficits and overall loss in connectivity strength [70]. This coincides with the second sharp decrease in the global FC signature occurred at $E Y O=0$. This is consistent with evidence from MRI studies that show volume loss accelerates near EYO $=0$ [24]. Together our data shows an overall pattern that initiates with a decline from a hyperactive state that briefly returns to baseline levels before declining near symptom onset. These results suggest a dual pathology process that reflects biological changes in the absence of behavioral changes. Understanding these distinct processes is clinically important for treatment intervention as individuals may respond differently based on their point on these curves.

The ability to characterize changes in a global FC signature during preclinical stages of AD was further supported by a separate linear model that focused on AT(N) biomarkers. A number of studies focusing on pathological biomarkers have proposed an AT(N) sequence 
in LOAD [60]. Our model for ADAD was given no a priori assumptions concerning the sequence of biomarker events, except that the sequence should be consistent for participants. Despite our assumption of no specific temporal sequence of biomarker progression, our work further supports that ADAD and LOAD have similar temporal patterns for $A D$ biomarkers regardless of the age of onset or genetic association [61]. Furthermore, we found disruption of the global FC signature was preferentially associated with both CSF $A \beta_{1-42}$ and hippocampal atrophy, biomarkers that characterize pathological changes occurring during the early and late stages of $A D$, respectively. This suggests that genetic mutations set in motion a timeline of $A T(N)$ biomarkers changes with brain amyloidosis (possibly, with certain soluble amyloid peptides proposed to be more closely associated with neuronal excitotoxicity) (A) followed by tauopathy (T) and eventually reduced glucose metabolism and brain volumetrics $(N)[6,7,62]$.

Our results contribute to the understanding of how, in AD etiology, changes in global network functionality precipitate eventual short-term episodic memory deficits that is the hallmark of AD. Based on these results it may be possible to use the global FC signature as a marker of underlying neuronal response to trials that introduce anti-A $\beta$ therapies very early in the disease. Advantages for FC as an outcome measure in clinical trials include lack of radiation unlike PET biomarkers, and being less invasive than a lumbar puncture for CSF. Further studies should also look at changes in comparison to LOAD. Longitudinal studies of changes in FC in this cohort are needed to more robustly evaluate the nature of neuronal dysfunction with disease progression.

\section{ACKNOWLEDGEMENTS}

Data collection and sharing for this project was supported by The Dominantly Inherited Alzheimer's Network (DIAN, UF1AG032438) funded by the National Institute on Aging (NIA), the German Center for Neurodegenerative Diseases (DZNE), Raul Carrea Institute for Neurological Research (FLENI), Partial support by the Research and Development, AMED, and the Korea Health Technology R\&D Project through the Korea Health Industry Development Institute (KHIDI). This manuscript has been reviewed by DIAN Study investigators for scientific content and consistency of data interpretation with previous DIAN Study publications. We acknowledge the altruism of the participants and their 
families and contributions of the DIAN research and support staff at each of the participating sites for their contributions to this study. Further support for this study was funded by NIH grants R01NR012907, R01NR012657, R01NR014449, P50AG05681, P01AG003991, P01AG026276, P30NS048056, UL1TR000448, R01AG04343404, R01AG052550, R01AG057680, K01AG053474, BrightFocus Foundation A2018817F and NSF grant DMS1300280. Funding was also provided by the Charles F. and Joanne Knight Alzheimer's Research Initiative, the Hope Center for Neurological Disorders, and generous support from the Fred Simmons and Olga Mohan Fund, the Paula and Rodger O. Riney Fund, and the Daniel J Brennan MD Fund. The authors would like to thank Drs. Abraham Snyder and Mathew Brier for their helpful suggestions. The authors would like to thank all the participants for their involvement in the study and DIAN sites for their recruitment and coordination of all studies.

\section{Author Contribution/Disclosure/Funding}

Robert X Smith: Primary author of the manuscript, developed the methodology conducted the analyses, and contributed to the interpretation of the data. Reports no disclosures. No Funding information to declare.

Jeremy F. Strain: Assisted in the conception and design of the study, consulted on the statistics, and reviewed the manuscript. Reports no disclosures. No Funding information to declare.

Aaron Tanenbaum: Assisted in the data analysis, statistics, and reviewed the manuscript. Reports no disclosures. No Funding information to declare.

Anne M. Fagan: Assisted in the data analysis, statistics, and reviewed the manuscript. Reports no disclosures. No Funding information to declare.

Jason Hassenstab: Assisted in the data analysis and reviewed the manuscript. Reports no disclosures. No Funding information to declare.

Eric McDade: Assisted in the original concept of the project, compiled the data, and reviewed the manuscript. Research support: NIA, Eli Lilly, Roche, Janssen, GHR Foundation; Advisory Board: Eli Lilly; DSMB: Eli Lilly. No Funding information to declare. 
Suzanne E. Schindler: Assisted in the data analysis and reviewed the manuscript. Reports no disclosures. No Funding information to declare.

Brian A. Gordon: Assisted in the original concept of the project, compiled the data, and reviewed the manuscript. Involved on a clinical trial sponsored by Avid. No Funding information to declare.

Chengjie Xiong: Assisted in statistical analyses and reviewed the manuscript. Reported no disclosures. No Funding information to declare.

Jasmeer Chhatwal: Compiled the data and reviewed the manuscript. Reported no disclosures. No Funding information to declare.

Clifford Jack Jr.: Compiled the data and reviewed the manuscript. Consults for Eli Lilly, and serves on an independent data monitoring board for Roche but receives no personal compensation from and commercial entity. He receives research support from NIH/NIA and the Alexander Family Professor of Alzheimer's Disease Research, Mayo Clinic. No Funding information to declare.

Celeste Karch: Compiled the data and reviewed the manuscript. Reports no disclosures. No Funding information to declare.

Sarah Berman: Compiled the data and reviewed the manuscript. Reports no disclosures. No Funding information to declare.

Jared R. Brosch: Compiled the data and reviewed the manuscript. Research support includes AbbVie, Avanir, Biogen, Eisai, Eli Lilly, Genetech, Novartis, Roche, Suven Life Sciences Lrd. No Funding information to declare.

James J. Lahl: Compiled the data and reviewed the manuscript. Reports no disclosures. No Funding information to declare.

Adam Brickman: Compiled the data and reviewed the manuscript. Reports no disclosures. No Funding information to declare.

David M. Cash: Compiled the data and reviewed the manuscript. Reports no disclosures. No Funding information to declare. 
Nick C. Fox: Compiled the data and reviewed the manuscript. Is on the scientific advisory board for Roche and Biogen. No Funding information to declare.

Neill R. Graff-Radford: Compiled the data and reviewed the manuscript. Research support includes AbbVie, Novartis, Biogen and Lilly. No Funding information to declare.

Johannes Levin: Compiled the data and reviewed the manuscript. Reports no disclosures. No Funding information to declare.

James Noble: Compiled the data and reviewed the manuscript. Reported no disclosures. No Funding information to declare.

David M. Holtzman: Compiled the data and reviewed the manuscript. Cofounded and is on the scientific advisory board of C2N diagnostics, LLC. Is on the scientific advisory board of Denali and consults for Genetech and AbbVie. No Funding information to declare.

Colin L. Masters: Compiled the data and reviewed the manuscript. Reports no disclosures. No Funding information to declare.

Martin R. Farlow: Compiled the data and reviewed the manuscript. Research support includes AbbVie, Accera, ADCS, Posiphen, Biogen, Eisai, Eli Lilly, Genentech, Novartis, Suven Life Sciences, Ltd. He is on the advisory boards for Accera, Allergan, Avanir, AZTherapies, Cognition Therapeutics, Cortexyme, Eli Lilly \& Company, Longeveron, Green Valley, Medavante, Merck and Co. Inc., Otsuka Pharmaceutical, Proclara, Neurotrope Biosciences, Regenera, Samumed, Takeda, vTv Therapeutics, Zhejian Hisun Pharmaceuticals. No Funding information to declare.

Christoph Laske: Compiled the data and reviewed the manuscript. Reports no disclosures. No Funding information to declare.

Peter R. Schofield: Compiled the data and reviewed the manuscript. Reports no disclosures. No Funding information to declare.

Daniel S. Marcus: Compiled the data and reviewed the manuscript. Funding support from Radiologics, Inc and WhiteRabbat.ai. No Funding information to declare. 
John C. Morris: Compiled the data and reviewed the manuscript. He is currently participating in clinical trials of antidementia drugs developed by Eli Lilly and Company, Biogen and Janssen. Dr. Morris serves as a consultant for Lilly USA. Research support from Eli Lilly/Avid Radiopharmaceuticals. No Funding information to declare.

Tammie L.S. Benzinger: Compiled the data and reviewed the manuscript. Involved in a clinical trial sponsored by Avid. No Funding information to declare.

Randall J. Bateman: Compiled the data and reviewed the manuscript. He is on the scientific advisory board for C2N Diagnostics. Research support from Abbvie, Biogen, Eisai, Eli Lilly, and Co/Avid Radiopharmaceuticals, Roche, Janssen, and United Neuroscience. No Funding information to declare.

Beau M. Ances: Compiled the data and reviewed the manuscript. Involved in a clinical trial sponsored by Avid. No Funding information to declare. 


\section{REFERENCES}

1. Amatniek JC, Hauser WA, DelCastillo-Castaneda C, Jacobs DM, Marder K, Bell K, et al., Incidence and predictors of seizures in patients with Alzheimer's disease. Epilepsia, 47(5):867-72 (2006).

2. Andersson JLR, Jenkinson $M$, Smith $S$, Non-linear registration, aka spatial normalisation. FMRIB technical report TR07JA2 (2010).

3. Afyouni S, Nichols TE, Insight and inference for DVARS, Neurolmage, 172:291-312 (2018).

4. Bateman RJ, Aisen PS, De Strooper B, et al. Autosomal-dominant Alzheimer's disease: a review and proposal for the prevention of Alzheimer's disease. Alzheimers Res Ther 2011;3:1-1.

5. Bateman RJ, Xiong C, Benzinger TL, et al. Clinical and biomarker changes in dominantly inherited Alzheimer's disease. N Engl J Med, 367:795-804 (2012).

6. Bateman RJ, Aisen PS, De Strooper B, et al. Autosomal-dominant Alzheimer's disease: a review and proposal for the prevention of Alzheimer's disease. Alzheimers Res Ther, 3:1-1 (2011).

7. Behzadi Y, Restom K, Liau J, Liu TT, A component based noise correction method (CompCor) for BOLD and perfusion based fMRI, Neurolmage, 37(1):90-101 (2007).

8. 47. Fischl B, FreeSurfer, Neurolmage, 62(2):774-781 (2012).

9. Benzinger TLS, Blazey T, Jack CR, Koeppe RA, Su Y, Xiong C, et al., Regional variability of imaging biomarkers in autosomal dominant Alzheimer's disease, Proc Natl Acad Sci USA, 110:e4502-e4509 (2013).

10. Berti V, Osorio RS, Mosconi L, Li Y, De Santi S, de Leon MJ, Early Detection of Alzheimer's Disease with PET Imaging, Neurodegener Dis, 7:131-135 (2010).

11. Brier MR, Gordon B, Friedrichsen K, McCarthy J, Stern A, Christensen J, et al. Tau and Abeta imaging, CSF measures, and cognition in Alzheimer's disease, Sci Transl Med, 8:338ra66 (2016).

12. Brier MR, Thomas JB, Snyder AZ, Benzinger TL, Zhang D, Raichle ME, et al., Loss of Intranetwork and Internetwork Resting State Functional Connections with Alzheimer's Disease Progression J Neurosci, 32(26):8890-8899 (2012). 
13. Brier MR, Thomas JB, Fagan AM, Hassenstab J, Holtzman DM, Benzinger TL, et al., Functional connectivity and graph theory in preclinical Alzheimer's disease, Neurobiology of Aging, 35(4):757-768 (2014).

14. Bobinski M, de Leon MJ, Wegiel J, Desanti S, Convit A, Saint Louis LA, et al., The histological validation of post mortem magnetic resonance imaging-determined hippocampal volume in Alzheimer's disease. Neuroscience, 95:721-5 (2000).

15. Buckner RL, Sepulcre J, Talukdar T, Krienen FM, Liu H, Hedden T, et al. Cortical hubs revealed by intrinsic functional connectivity: mapping, assessment of stability, and relation to Alzheimer's disease, J Neurosci, 29: 1860-73 (2009).

16. Burdick D, Soreghan B, Kwon M, Kosmoski J, Knauer M, Henschen A, et al., Assembly and

17. aggregation properties of synthetic Alzheimer's A4/beta amyloid peptide analogs. The Journal of Biological Chemistry, 267, 546-554 (1992).

18. Chhatwal JP, Schultz AP, Johnson K, Benzinger TLS, Jack C, Ances BM, et al., Impaired default network functional connectivity in autosomal dominant Alzheimer disease, Neurology, 81(8):736-744 (2013).

19. Chhatwal JP, Schultz AP, Johnson KA, Hedden T, Jaimes S, Benzinger TLS, et al., Preferential degradation of cognitive networks differentiates Alzheimer's disease from ageing, Brain, 141:1486-500 (2018).

20. Cirrito, JR, et al., In vivo assessment of brain interstitial fluid with microdialysis reveals plaque-associated changes in amyloid- $\beta$ metabolism and half-life, J Neurosci 23:8844-8853 (2003).

21. Cirrito HR, Yamada KA, Finn MB, Sloviter RS, Bales KR, May PC, Schoepp DD, Paul SM, Mennerick S, Holtzman DM, Synaptic activity regulates interstitial fluid amyloid- $\beta$ levels in vivo, Neuron, 48:913-922 (2005).

22. Dennis JE, Schnabel RB, Numerical Methods for Unconstrained Optimization and Nonlinear Equations. Prentice-Hall, Englewood Cliffs, NJ (1983).

23. Dubois B, Hampel H, Feldman HH, et al. Preclinical Alzheimer's disease: definition, natural history, and diagnostic criteria. Alzheimers Dement,12:292-323 (2016). 
24. Ewers M, Sperling RA, Klunk WE, Weiner MW, Hampel H, Neuroimaging markers for the prediction and early diagnosis of Alzheimer's disease dementia, Trends in Neurosciences, 34(8):430-442 (2011)

25. Fennema-Notestine C, et al., Alzheimer's Disease Neuroimaging Initiative, Structural MRI biomarkers for preclinical and mild Alzheimer's disease, Hum Brain Mapp 30(10):3238-3253 (2009).

26. Forsberg A, Engler H, Almkvist O, Blomquist G, Hagman G, Wall A, et al., PET imaging of amyloid deposition in patients with mild cognitive impairment, Neurobiology of Aging, 29(10):1456-1465 (2008).

27. Franzmeier N, Koutsouleris N, Benzinger T, et al. Predicting sporadic Alzheimer's disease progression via inherited Alzheimer's disease-informed machinelearning. Alzheimer's Dement 2020; 16: 501- 511.

28. Frisoni GB, Alzheimer disease: biomarker trajectories across stages of Alzheimer disease, Nat Rev Neurol, 8:299-300 (2012).

29. Frisoni GB, Fox NC, Jack Jr CR, Scheltens PS, Thompson PW, The clinical use of structural MRI in Alzheimer disease, Nature Reviews Neurology, 6:67-77 (2010).

30. Frost B, Diamond MI, Prion-like mechanisms in neurodegenerative diseases, Nature Reviews Neuroscience, 11:155-159 (2010).

31. Gholipour A, Kehtarnavaz N, Gopinath K, Briggs R, Panahi I, Average field map image template for Echo-Planar image analysis. Conf Proc IEEE Eng Med Biol Soc, 2008:94-97 (2008).

32. Gordon BA, Blazey TM, Su Y, Hari-Raj A, Dincer A, Flores S, et al., Spatial patterns of neuroimaging biomarker change in individuals from families with autosomal dominant Alzheimer's disease: a longitudinal study, Lancet Neurol, 17(3):241-250 (2018).

33. Greicius MD, Krasnow B, Reiss AL, Menon V. Functional connectivity in the resting brain: a network analysis of the default mode hypothesis. Proc Natl Acad Sci USA, 100:253-8 (2003).

34. Greicius MD, Srivastava G, Reiss AL, Menon V, Default-mode network activity distinguishes Alzheimer's disease from healthy aging: evidence from functional MRI, Proc Natl Acad Sci USA, 101:4637-42 (2004). 
35. Huang $Y$, Potter R, Sigurdson W, et al. Effects of Age and Amyloid Deposition on $A \beta$ Dynamics in the Human Central Nervous System, Arch Neurol, 69(1):51-58 (2012).

36. Jack CR, Knopman DS, Weigand SD, Wiste HJ, Vemuri P, Lowe V, Kantarci K, Gunter JL, Senjem ML, Ivnik RJ, Roberts RO, Rocca WA, Boeve BF, and Petersen RC, An operational approach to National Institute on Aging-Alzheimer's Association criteria for preclinical Alzheimer disease. Ann Neurol, 71:765-775 (2012).

37. Jack CR, Knopman DS, Jagust WJ, Shaw LM, Aisen PS, Weiner MW, Petersen RC, Trojanowski JQ, Hypothetical model of dynamic biomarkers of the Alzheimer's pathological cascade, Lancet Neurol, 9:119-128 (2010).

38. Jenkinson M, Bannister PR, Brady JM, Smith SM, Improved optimisation for the robust and accurate linear registration and motion correction of brain images. Neurolmage, 2002;17(2):825-841.

39. Klunk WE, Engler H, Nordberg A, Wang Y, Blomqvist G, Holt DP, et al., Imaging brain amyloid in Alzheimer's disease with Pittsburgh Compound-B, Ann Neurol, 55:306319 (2004).

40. Kinnunen KM, Cash DM, Poole T, Frost C, Benzinger TLS, Ahsan RL, Leung KK, Cardoso MJ, Modat M, Malone IB, Morris JC, Bateman RJ, Marcus DS, Goate A, Salloway SP, Correia S, Sperling RA, Chhatwal JP, Mayeux RP, Brickman AM, Martins RN, Farlow MR, Ghetti B, Saykin AJ, Jack Jr CR, Schofield PR, McDade E, Weiner MW, Ringman JM, Thompson PM, Masters CL, Rowe CC, Rossor MN, Ourselin S, Fox NC, Presymptomatic atrophy in autosomal dominant Alzheimer's disease: A serial magnetic resonance imaging study. Alzheimers Dement, 14:43-53 (2018).

41. Levene $H$, Robust tests for equality of variances, In Ingram Olkin; Harold Hotelling; et al. Contributions to Probability and Statistics: Essays in Honor of Harold Hotelling. Stanford University Press, 278-292 (1960).

42. Mattsson N, Insel PS, Palmqvist S, Stomrud E, van Westen D, Minthon L, Zetterberg $\mathrm{H}$, Blennow K, and Hansson O, Increased amyloidogenic APP processing in APOE ع4negative individuals with cerebral $\beta$-amyloidosis. Nat Commun 7:10918 (2016).

43. McDade E, Wang G, Gordon BA, Hassenstab J, Benzinger TLS, Buckles V, et al., Longitudinal cognitive and biomarker changes in dominantly inherited Alzheimer disease, Neurology, 91(14):e1295-e1306 (2018). 
44. McKhann G, Drachman D, Folstein M, Katzman R, Price D, Stadlan EM, Clinical diagnosis of Alzheimer's disease, Neurology, 34(7):939 (1984).

45. Mendez M, Lim G, Seizures in elderly patients with dementia: epidemiology and management. Drugs Aging, 20(11):791-803 (2003).

46. Mintun MA, Larossa GN, Sheline YI, Dence CS, Lee SY, Mach RH, et al. [11C]PIB in a nondemented population: potential antecedent marker of Alzheimer disease, Neurology, 67:446-452 (2006).

47. Mosconi L, Brain glucose metabolism in the early and specific diagnosis of Alzheimer's disease, Eur J Nucl Med Mol Imaging, 32:486 (2005).

48. Mosconi L, Berti V, Glodzik L, Pupi A, De Santi S, de Leon MJ, Pre-Clinical Detection of Alzheimer's Disease Using FDG-PET, with or without Amyloid Imaging, J Alzheimer's Disease, 20(3):843-854 (2010).

49. Morris JC, The Clinical Dementia Rating (CDR): Current version and scoring rules, Neurology, 43(11), 2412-2414 (1993).

50. Ovsepian SV, O'Leary VB, Neuronal activity and amyloid plaque pathology: an update. J Alzheimers Dis, 49(1):13-9. (2016).

51. Palop JJ, Chin J, Roberson ED, Wang J, Thwin MT, Bien-Ly N, Yoo J, Ho KO, Yu GQ, Kreitzer A, Finkbeiner S, Noebels JL, Mucke L, Aberrant excitatory neuronal activity and compensatory remodeling of inhibitory hippocampal circuits in mouse models of Alzheimer's disease. Neuron, 55:697-711 (2007).

52. Palop JJ, Mucke L, Epilepsy and cognitive impairments in Alzheimer disease. Arch Neurol, 66(4):435-40 (2009).

53. Power JD, Barnes KA, Snyder AZ, Schlaggar BL, Petersen SE. Spurious but systematic correlations in functional connectivity MRI networks arise from subject motion. Neuroimage, 59: 2142-54 (2012).

54. Power JD, Cohen AL, Nelson SM, et al., Functional network organization of the human brain. Neuron, 72(4):665-78 (2011).

55. Preische O, Schultz SA, Apel A, Kuhle J, Kaeser SA, Barro C, Gräber S, Kuder-Buletta E, LaFougere C, Laske C, Vöglein J, Levin J, Masters CL, Martins R, Schofield PR, Rossor MN, Graff-Radford NR, Salloway S, Ghetti B, Ringman JM, Noble JM, Chhatwal J, Goate AM, Benzinger TLS, Morris JC, Bateman RJ, Wang G, Fagan AM, 
McDade EM, Gordon BA, Jucker M; Dominantly Inherited Alzheimer Network. Nat Med, 25(2):277-283 (2019).

56. $\mathrm{R}$ Core Team, $\mathrm{R}$ : A language and environment for statistical computing. $\mathrm{R}$ Foundation for Statistical Computing, Vienna, Austria. URL https://www.Rproject.org/ (2017).

57. Rousset OG, Collins DL, Rahmim A, Wong DF Design and implementation of an automated partial volume correction in PET: application to dopamine receptor quantification in the normal human striatum. J Nucl Med, 49:1097-1106 (2008).

58. Ryman DC, Acosta-Baena N, Aisen PS, Bird T, Danek A, Fox NC, et al., Symptom onset in autosomal dominant Alzheimer disease, Neurology, 83(3):253-260 (2014).

59. Schindler SE, Fagan AM, Autosomal dominant Alzheimer disease: a unique resource to study CSF biomarker changes in preclinical AD, Frontiers in neurology, 6:142 (2015).

60. Schnabel RB, Koontz JE, Weiss BE, A modular system of algorithms for unconstrained minimization. ACM Transactions on Mathematical Software, 11:419440 (1985).

61. Schultz AP, Chhatwal JP, Hedden T, Mormino EC, Hanseeuw BJ, Sepulcre J, Huijbers W, LaPoint M, Buckley RF, Johnson KA, Sperling RA, Phases of Hyperconnectivity and Hypoconnectivity in the Default Mode and Salience Networks Track with Amyloid and Tau in Clinically Normal Individuals, J Neuroscience, 37(16):4323-4331 (2017).

62. Shapiro SS, Wilk MB, An analysis of variance test for normality (complete samples), Biometrika, 52 (3-4): 591-611 (1965)

63. Smailagic N, Vacante M, Hyde C, Martin S, Ukoumunne O, Sachpekidis C, 18F-FDG PET for the early diagnosis of Alzheimer's disease dementia and other dementias in people with mild cognitive impairment (MCI), Cochrane Database Syst Rev 1 (2015).

64. Smith SM, Jenkinson M, Woolrich MW, Beckmann CF, Behrens TEJ, Johansen-Berg $H$, et al., Advances in functional and structural MR image analysis and implementation as FSL, 23:208-219 (2004). 
65. Smith RX, Jann K, Dapretto M, Wang DJJ, Imbalance of Functional Connectivity and Temporal Entropy in Resting-State Networks in Autism Spectrum Disorder: A Machine Learning Approach, Front Neurosci, 12: 869 (2018).

66. Su Y, D'Angelo GM, Vlassenko AG, Zhou G, Snyder AZ, Marcus DS, et al. Quantitative analysis of PiB-PET with FreeSurfer ROIs. PLoS One 2013;8: e73377.

67. Guangyu Cm Hao Sm Gang Cm Douglas WB., et al. Staging Alzheimer's Disease Risk by Sequencing Brain Function and Structure, Cerebrospinal Fluid, and Cognition Biomarkers. JAD. 54, 983-993 (2016).

68. Palop JJ, Mucke L. Amyloid-beta-induced neuronal dysfunction in Alzheimer's disease: From synapses toward neural networks. Nat Neurosci 13, 812-818 (2010).

69. Su Y, Blazey TM, Snyder AZ, Raichle ME, Marcus DS, Ances BM, et al. Partial volume correction in quantitative amyloid imaging. Neuroimage, 107:55-64 (2015).

70. Thomas JB, Brier MR, Bateman RJ, Snyder AZ, Benzinger TL, Xiong C, et al., Functional Connectivity in Autosomal Dominant and Late-Onset Alzheimer Disease, JAMA Neurology, 71(9):1111-1122 (2014). 


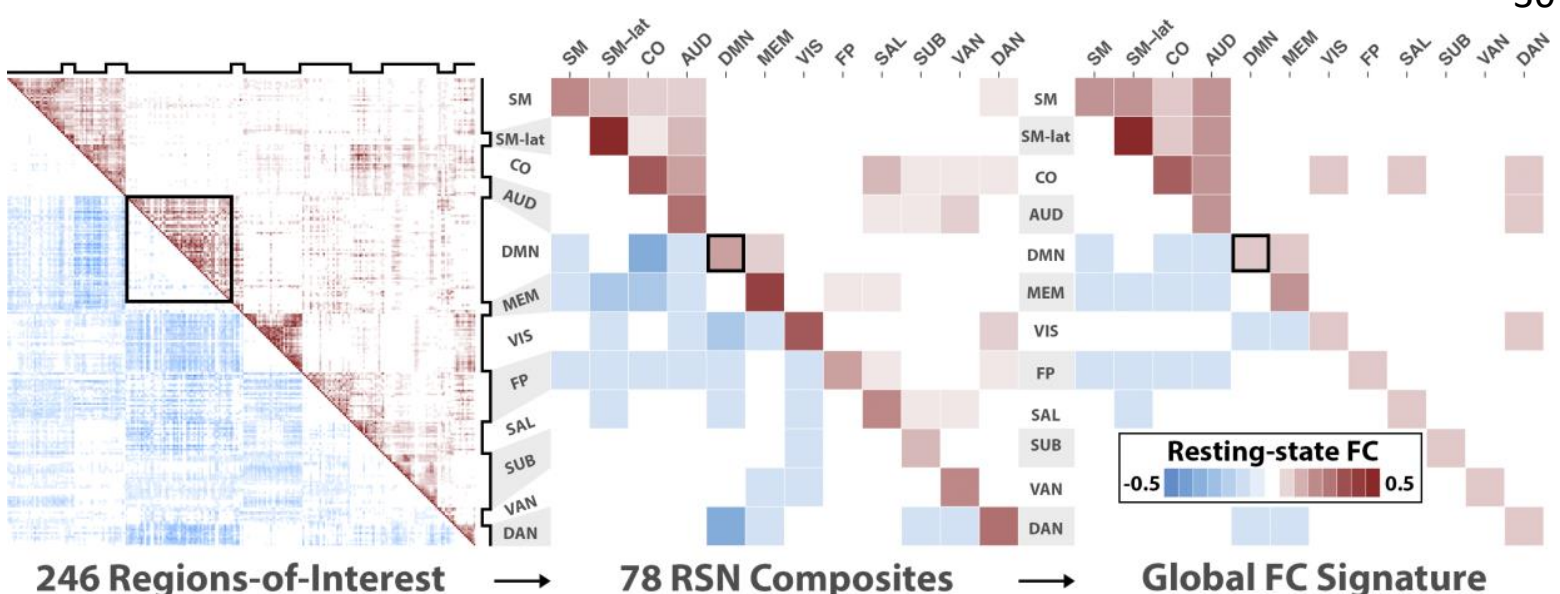

Figure 1. Principal component analysis (PCA) reveals a distributed pattern of functional connectivity (FC) changes across cortical and subcortical resting state networks (RSNs). LEFT: The mean FC matrix across all participants. An FC matrix from 248 regions of interest was computed for each participant. Positive correlations are shown in the upper triangle, and negatives are shown in the bottom triangle. Notched black lines on the top and to the right indicate RSNs. The black box outline is a visual aid highlighting the intra-network FC values of the default mode network (DMN). MIDDLE: The mean FC-composite matrix across all participants. For each participant an FC-composite matrix was generated by computing the mean intra- and inter-network FC matrix values (total of 78) for all 12 RSNs. Here the black box highlights the mean intra-network FC value of the DMN. RIGHT: The global FC signature is derived from the primary PCA pattern and reflects positively and negatively weighted mean FC-composite values. The strongest positive weights include the sensorimotor (SM, SMlat), cingulo-opercular (CO), auditory (AUD), visual (VIS), and memory (MEM). The strongest negative weights included the fronto-parietal (FP), MEM, dorsal attention (DAN), and default mode (DMN). The black box outline highlights the weight of the mean intra-network FC value of the DMN. 


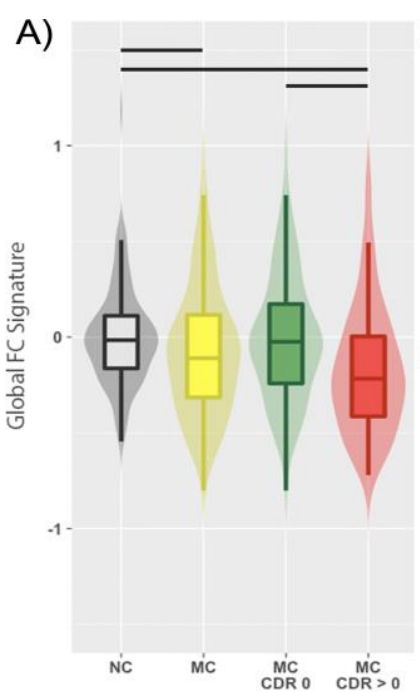

B)

C)

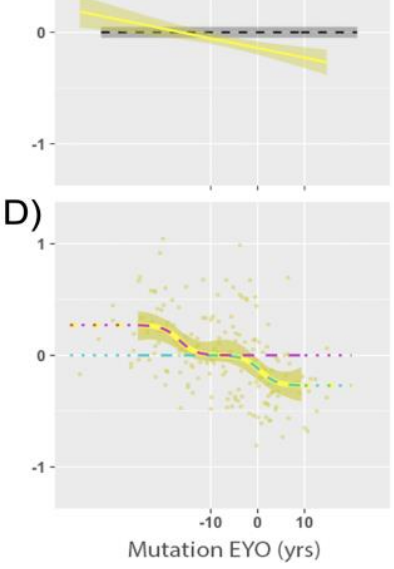

Figure 2. A) The global functional connectivity (FC) signature as a functional of mutation status, clinical dementia rating (CDR) and expected years to symptom onset (EYO). A) Violin and box plots of the global FC signature for NC (gray) and MC (yellow), MC CDR 0 (green), and MC CDR $>0$ (red) participants. NC had a higher global FC signature compared to $M C$. MC CDR>0 had significant decreases in the global FC signature compared to NC and MC CDR 0 participants. A black bars represents a significant group difference. B) Line plot showing the association between global FC signature and mutation EYO for MC participants. The global FC signature was associated with EYO in MC $(p<0.05)$. The dashed line (black) is the mean global FC signature in NC participants, and the dark gray band is the confidence interval defined as two standard errors of the mean. C) Coefficient of determination $\left(R^{2}\right)$ for the global FC signature and the intra-network values of 12 RSNs. The length of the vertical bar represents the strength of that FC value for predicting EYO. D) The global FC signature for MC individuals exhibits a biphasic behavior with regards to mutation EYO in \pm 5 -year bins. When the global FC signature was fit to the bin means (yellow curve), two logistic curves were observed (magenta, and cyan). A two stage process was observed, with early and late changes seen in the global FC signature. 


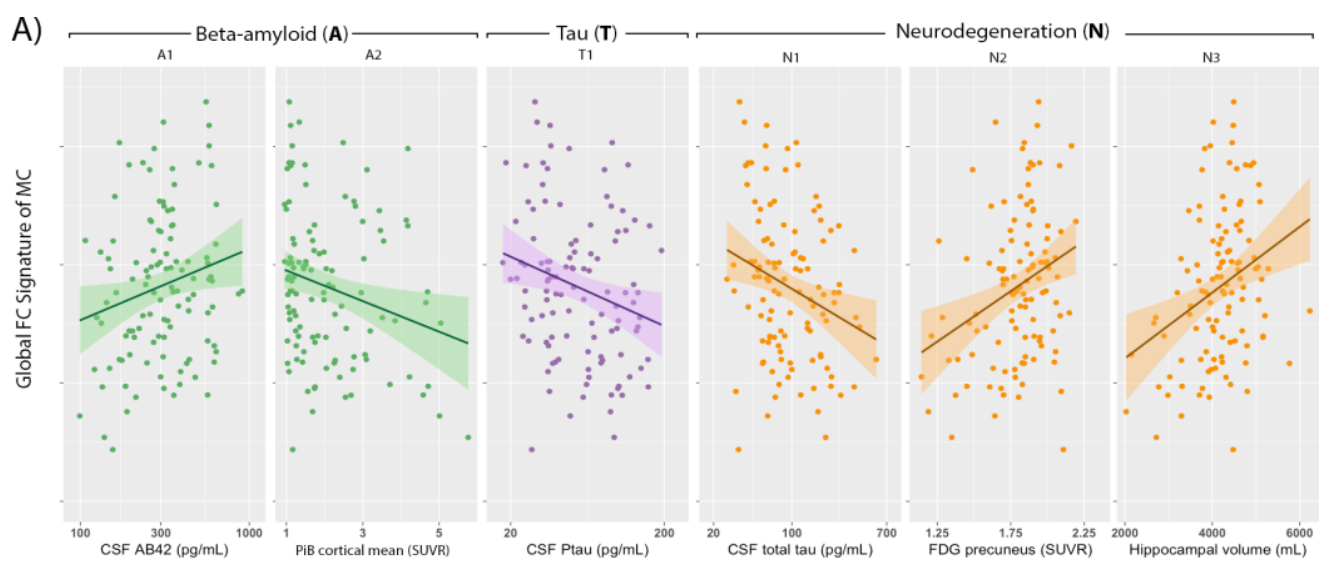

B)
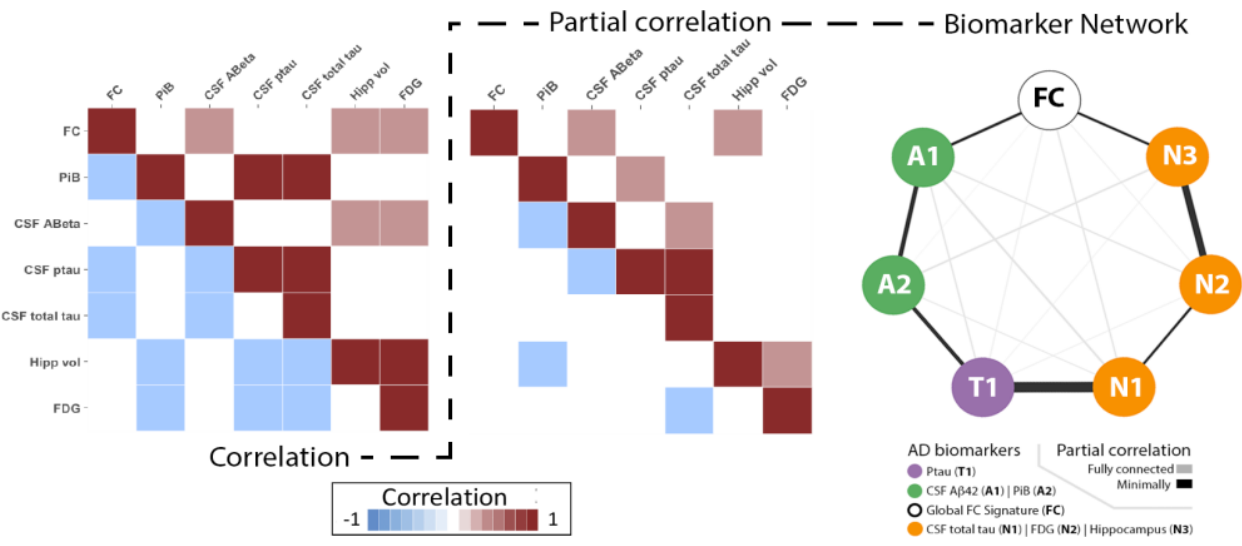

Figure 3. The global FC signature as a function of Alzheimer disease (AD) biomarkers in mutation positive (MC) individuals. Analyses investigated amyloid ( $A$; green), Tau (T; purple), and neurodegenerative ( $\mathrm{N}$; orange) biomarkers. Scatter plots show the global FC signature was $A$ ) positively associated with cerebrospinal fluid (CSF) $A \beta_{1-42}$, negatively associated with mean Pittsburgh B (PiB) standardized uptake value ratios (SUVR), negatively associated with CSF phosphorylated tau 181 ( $p$-tau $\left.\tan _{181}\right)$ and total tau, and positively associated with both precuneus fluorodeoxyglucose (FDG) uptake and hippocampal volume. B) The correlation matrix of all biomarkers, including global FC signature (left), was inverted to compute the partial correlation matrix (middle). Network map of relationships was plotted among the global FC signature and AD biomarkers based on the partial correlation matrix. The fully (light edges) and minimally (dark edges) connected graphs are shown. All associations were corrected for age. 
Table 1

\begin{tabular}{|c|c|c|}
\hline & NC & MC \\
\hline $\mathrm{N}$ & 104 & 171 \\
\hline Age: $\mathrm{M}(\mathrm{SD})$ & $40.2(11.3)$ & $39.4(11.3)$ \\
\hline Gender (M/F) & $41 / 63$ & $74 / 97$ \\
\hline CDR (0/0.5/>0.5) & $104 / 0 / 0$ & $99 / 46 / 26$ \\
\hline
\end{tabular}

NC $=$ Non-Carrier

$\mathrm{MC}=$ Mutation Carrier

$\mathrm{CDR}=$ Clinical Dementia Ratio 